\title{
Load Shedding Controllers against Voltage Instability: a comparison of designs
}

\author{
C. Moors \\ D. Lefebvre \\ T. Van Cutsem
}

\begin{abstract}
This paper discusses and compares three types of undervoltage load shedding closed-loop controllers in terms of performances and design computational effort. We first describe the various controllers and iden tify the parameters to be optimized. Next, w epresent an optimization methodology applicable to all three types of controllers. This approach allows to find the controller parameters which optimize an ov erall performance objective. Results are presented on the Hydro-Québec system, in which load shedding is presently planned.
\end{abstract}

Keywords - Voltage stability, system protection schemes, load shedding, combinatorial optimization

\section{INTRODUCTION}

$I^{\mathrm{N}}$ the deregulated en vironment and owing to the difficult y of building new transmission and generation facilities, po w er systems will be operated closer to their stability limits. There are tw o lines of defence against incidents likely to trigger such instabilities:

- preventively: estimate securit y margins with respect to credible contingencies, i.e. incidents with a reasonable probability of occurrence, and take appropriate preventive actions to restore sufficient margins when needed;

- correctively. implement automatic correctiv eactions, through System Protection Schemes (SPS), to face the more severe, but less likely incidents [1], [2].

Preventive security criteria usually require that the system remains stable after any credible contingency, without the help of correctiv eactions. The main reason is that these actions usually affect generators and/or loads, which is acceptable only in the presence of severe disturbances.

The present paper concentrates on long-term voltage instabilit y driven by Load Tap Changers (LTCs), generator OverExcitation Limiters (OELs), switched shunt compensation, restorative loads, and possibly secondary voltage control. This type of instability has become a major threat in many systems [3], [4].

Since long-term voltage instability is triggered mainly by the loss of generation or transmission facilities, "N-1" contingencies corresponding to the loss of a single equipment are usually considered in preventive securit y analysis. On the other hand, N-2 and more severe disturbances should be counteracted by an SPS. While it must be used in the last resort and to the least extent, automatic load shedding is very effective in this respect.

C. Moors (moors@montefiore.ulg.ac.be) and T. V an Cutsem (vct@montefiore.ulg.ac.be) are with the Dept. of Electrical Engineering (Montefiore Institute) of the University of Liège, Sart Tilman B37, B-4000 Liège, Belgium

C. Moors is a F.R.I.A. researcher; T. Van Cutsem is a research director of F.N.R.S

D. Lefebvre (lefebvre@rti.h ydro.qc.ca) is with the Ilansenergie Division of Hydro-Québec, CP 10000 Montréal (QC), Canada
A few undervoltage load shedding schemes have been proposed or implemented throughout the w orld(e.g. [1], $[5],[6],[7],[8])$. This paper compares three types of closedloop load shedding controllers in terms of performances and design computational effort. The first $t \mathrm{w}$ o cotrollers are local by nature: they rely on local measurements taken from the system and shed loads once the observed signal stays below some threshold for some time. The third controller is a step tow ards a wide-area protection, in the sense that other post-disturbance correctiv econtrols are managed in addition to load shedding.

The paper is organized as follows. Section II describes the common structure of the various controllers, while Section III explains how their various parameters are optimized. Section IV compares the performances of the controllers on a rather complex example taken from the HydroQuébec system, in which an undervoltage load shedding scheme is planned. Conclusions and perspectives are presented in Section V.

\section{STRUCTURE OF THE CONTROLLERS}

All protections considered in this paper rely on a measured signal which is typically the average voltage $V$ over several transmission buses in the load area of concern. Other measurements could also enter the logic, such as the reactiv e reserv e of neighouring generators, etc.Individual loads are shed according to a predefined bus ranking, determined as outlined is section III.a. It must be emphasized that each protection operates in closed-loop since $V$ is contin uously measured and the protection may trigger several, successive load sheddings, if needed.

\section{A. Fixed-Steps Fixed Delays (FSFD) controller.}

The first type of controller relies on $k$ rules of the type:

$$
\text { if } V<V_{i}^{\text {min }} \text { during } d_{i} \text { seconds, shed } \Delta P_{i} M W
$$

The number $k$ of rules is decided a priori; in practice it is typically equal to 2 or 3 .

A two-rule example from the Hydro-Qubec system is given in Fig. 1, in which a star indicates a shunt compensation switching and $\mathrm{Rx}$ a load shedding due to rule Rx. As can be seen, the total shedding results from tw o firings of $R_{2}$, follow ed $\mathrm{b}$ one firing of $R_{1}$.

Note also that the above rules are "concurrent". In the case of Fig. 1, for instance, both rules have their "if clause" satisfied just after the disturbance. Due to its much larger timing, $R_{1}$ is not fired before being reset (at $t=12 \mathrm{~s}$ ) under the effect of $R_{2}$. 


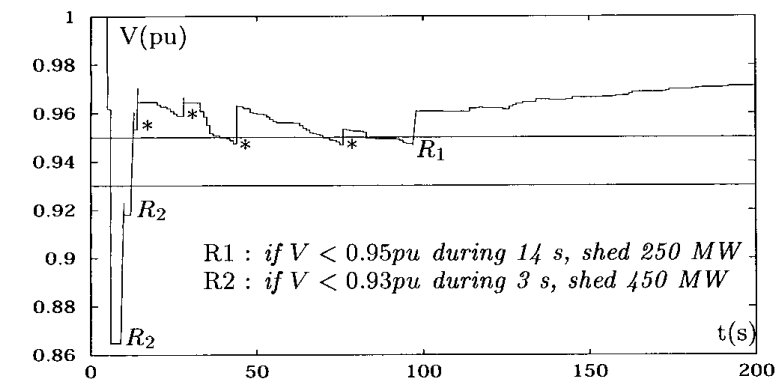

Fig. 1. 2-rule protection example from the Hydro-Québec system

The optimization of such a controller consists in properly determining the $3 k$-dimensional vector of unknows:

$$
\mathbf{x}=\left[V_{1}^{m i n}, d_{1}, \Delta P_{1}, \ldots, V_{k}^{m i n}, d_{k}, \Delta P_{k}\right]
$$

Clearly, it is required to adjust the amount of load shedding to the severity of the situation. To meet this objective, the above controller must be provided with either a very fast acting rule (which may interact with the other rules) or additional rules (which significantly increase the computational burden of the design). This has motivated the use of the next design.

\section{B. Variable-Step Variable Delay (VSVD) controller.}

The second type of controllers is a kind of generalization of the previous one. It relies on a unique rule whose activation delay $d$ and amount of shed load $\Delta P$ depend on the time evolution of $V$. The idea is to set up a controller adjusting its action automatically to the severity of the situation it is facing: the faster the decrease in $V$, the shorter $d$ and the larger $\Delta P$.

Thus, the delay is determined by the following inversetime characteristic:

$$
\int_{t_{0}}^{t_{0}+d}\left(V^{m i n}-V\right) d t=C \quad \text { with } \quad d_{\min } \leq d \leq d_{\max }
$$

where $t_{0}$ is the time at which $V$ becomes smaller than $V^{\text {min }}$ and $C$ is a constant to be optimized at the design stage.

In the same manner, the load shedding step is given by :

$$
\Delta P=k \Delta V_{a v g} \text { with } \quad \Delta P_{\min } \leq \Delta P \leq \Delta P_{\max }
$$

where $\Delta V_{a v g}$ is the average voltage drop over the $\left[t_{0} t_{0}+d\right]$ interval:

$$
\Delta V_{a v g}=\frac{1}{d} \int_{t_{0}}^{t_{0}+d}\left(V^{\min }-V\right) d t
$$

Two variants of this controller have been considered: - the Variable-Step Fixed Delay (VSFD) controller works with a constant delay $d$ and sheds load according to (3).

- in the Fixed-Step Variable Delay (FSVD) controller, the delay is adjusted using (2) while the shedding step $\Delta P$ is constant.

The optimization requires the determination of one of the following vectors, depending on the variant considered:

$$
\begin{aligned}
& \mathbf{x}_{\mathrm{VSVD}}=\left[V^{\min }, C, d_{\min }, d_{\max }, k, \Delta P_{\min }, \Delta P_{\max }\right] \\
& \mathbf{x}_{\mathrm{FSVD}}=\left[V^{\min }, C, d_{\min }, d_{\max }, \Delta P\right] \\
& \mathbf{x}_{\mathrm{VSFD}}=\left[V^{\min }, d, k, \Delta P_{\min }, \Delta P_{\max }\right]
\end{aligned}
$$

\section{Coordinated controller.}

As is well-known, several post-disturbance actions are possible to counteract voltage instability. Among them, shunt compensation switching is the first to come to mind. In the example of Fig. 1, the pre-existing shunt reactor tripping scheme has been taken into account when optimizing the settings of the two-rule controller. This optimization has implicitely taken the most benefit from the imposed compensation.

In the last type of controllers, a more general viewpoint is adopted by coordinating load shedding together with the other post-disturbance corrective controls, thereby approaching a notion of wide-area protection.

The logic is based on several rules. The first one relates to load shedding and is the same as for the VSVD, VSFD or FSVD controller. The other rules are associated to the other corrective controls managed by the protection. In this work, we have considered shunt compensation switching in addition to load shedding. The following rule is used to decide the switching of the compensations:

\section{if $V<V^{\text {sh }}$ during $\delta$ (or $\left.\delta^{\prime}\right)$ seconds, switch one more shunt}

Preliminary simulations showed that the controller should trip shunt reactors as soon as possible in order to minimize the amount of shed load. Therefore, we introduced two delays. The $\delta^{\prime}$ delay relates to the (short) time period between two successive switchings, while the $\delta$ parameter refers to the (larger) delay preceding the first action. The value of the latter has to be carefully chosen in order the controller not to act when facing any temporary voltage drops. In this logic, we have also to determine the location where the compensation has to be switched. To this purpose, each time the rule has to be fired we check the transmission bus voltages and we switch compensation at the bus with the lowest voltage.

The optimization of this controller requires the determination of the $V^{s h}, \delta$ and $\delta^{\prime}$ parameters together with the ones relative to the load shedding rule (4), (5) or (6).

\section{DESIGN OF THE CONTROLLERS}

The methodology used to adjust the settings of the various controllers consists of two steps. In the first step, a set of training scenarios is built, and each unstable scenario of this set is analyzed to determine the minimal load shedding which stabilizes the system. In the second step, the protection parameters are determined in order to approach as closely as possible the optimal sheddings computed in the first step, over the whole set of scenarios. A "clever" enumerative optimization method is used to this purpose.

\section{A. Scenario analysis}

The first step of our approach consists in setting up a set of $s$ training scenarios, corresponding to various topologies, load levels, generation schemes, contingencies, etc.

In principle all the scenarios to be dealt with by a single protection should involve the same weak area of the 
system. In other words, the instability modes and hence the optimal shedding locations should be rather close for all the unstable scenarios of the set. Therefore, we assume that a common bus ranking can be set up, e.g. through eigenvector analysis, as described in [9]. Once this ranking has been identified, the minimal amount of load shedding $P_{i}^{\star}(\mathrm{i}=1, \ldots, \mathrm{s})$ is determined for each scenario.

It must be emphasized that the $P_{i}^{\star}$ values depend on the (automatic) shunt compensation switching sequences. When optimizing the FSFD or VSVD controllers, the settings of the compensation control devices are fixed (at their existing values); therefore, $P_{i}^{\star}$ can be determined by varying the amount and delay of load shedding, using the procedure described in [9]. On the other hand, when optimizing the coordinated controller, shunt compensation becomes itself a degree of freedom, which makes the determination of $P_{i}^{\star}$ more complex. To keep the problem tractable, we have made the reasonable assumption that the faster the shunt compensation switching, the lower the minimal amount to shed. The $P_{i}^{\star}$ values are therefore computed by assuming that the whole compensation is switched just after the disturbance occurrence.

\section{B. Statement of the design problem}

Given the $s$ training scenarios, the problem is to determine the vector of unknowns $\mathbf{x}$ relative to the controller of concern, such that the following requirements are met:

1. the amount of load shedding must be as close as possible to the minimum $P_{i}^{\star}$ determined in the first step;

2. all unstable scenarios must be saved (dependability);

3. no load must be shed in a stable scenario (security);

4. optionally, some other constraints can be imposed. For instance, the distribution voltages should not stay below some threshold for more than some time.

This can be translated into an optimization problem: minimize the discrepancies $P_{i}^{s h}(\mathbf{x})-P_{i}^{\star}$, where $P_{i}^{s h}(\mathbf{x})$ is the total load power shed in the $i$-th scenario (a function of $\mathbf{x}$ ). Among the possible objective functions, we pay attention to the $L_{1}$ norm:

$$
\min _{\mathbf{x}} \frac{1}{s} \sum_{i}\left[P_{i}^{s h}(\mathbf{x})-P_{i}^{\star}+p_{i}(\mathbf{x})\right]
$$

as well as the $L_{\infty}$ norm:

$$
\min _{\mathbf{x}} \max _{i}\left[P_{i}^{s h}(\mathbf{x})-P_{i}^{\star}+p_{i}(\mathbf{x})\right]
$$

where the sum and the max extend over the unstable scenarios and $p_{i}(\mathbf{x})$ is a penalty term accounting for the violation of the above requirements. Note that in (7) and (8), the expression within brackets is always positive since $P_{i}^{s h}>P_{i}^{\star}$ and $p_{i}(\mathbf{x}) \geq 0$.

The penalties are chosen as follows:

- when the system is unstable (requirement 2 violated), transmission voltages eventually become smaller than some threshold $V_{\text {low }}$. Denoting by $t_{\text {low }}$ the time at which this occurs, the penalty takes on the form:

$$
p_{i}=\frac{C_{1}}{t_{l o w}+C_{2}} \quad C_{1} \gg 0 \quad C_{2}>0
$$

- when an amount $P_{i}^{s h}$ is shed in a stable case (requirement 3 violated), the penalty term takes on the form:

$$
p_{i}=C_{3} P_{i}^{s h} \quad C_{3} \gg 1
$$

- let $t_{r e c}$ be the recovery time, i.e. the time at which voltages are again larger than a specified value. Requirement 4 consists in specifying that $t_{r e c}$ is smaller than a given value $t_{r e c}^{\max }$. If this does not hold, the penalty is taken as:

$$
p_{i}=C_{4}\left(t_{r e c}-t_{r e c}^{\max }\right) \quad C_{4} \gg 0
$$

Note that with the above penalties, the more dangerous a situation (i.e. the shorter $t_{\text {low }}$ or the larger $t_{\text {rec }}$ ), the higher the penalty. This is expected to provide the optimization method with information on how to improve the parameters.

\section{The branch-and-bound approach}

The optimization problem (7-11) is complex. Indeed, both $P_{i}^{s h}$ and $p_{i}$ must be determined from time-domain simulations and hence, explicit analytical expressions cannot be established. Moreover, they vary with $\mathbf{x}$ in a discontinuous manner, which prevents from using analytical optimization methods. Finally, multiple local minima are expected. This is why we prefer to resort to combinatorial optimization.

To this purpose, each component of $\mathbf{x}$ is discretized in a finite number of possible values. The discretization steps are chosen in accordance with the engineering knowledge of the problem. For instance, the voltage steps are compatible with measurement accuracy, the lower bound on shedding steps is the minimal amount that can be tripped by opening distribution feeders, etc.

A brute-force approach would consist in evaluating the objective function for all possible values of $\mathbf{x}$ and selecting the best one as solution of the problem, as detailed in the algorithm in Fig. 2 (please ignore the bold type line for the time being).

Note that the evaluation of $L_{1}$ or $L_{\infty}$ for a given protection setting requires the simulation of the s scenarios in order to compute the s discrepancies $P_{i}^{s h}(\mathbf{x})-P_{i}^{\star}+p_{i}(\mathbf{x})$, which is very time consuming. Accordingly, this approach cannot be envisaged in real-life problems.

However, this brute force enumeration can be significantly improved in the following way. In the above algorithm, during the enumeration we keep track of the best objective $B$ reached so far. This value $B$ is an upper bound on the sought global minimum. Therefore, in the course of computing the $L_{1}$ or $L_{\infty}$ objective, as soon as the latter becomes greater than $B$, we can skip the current value of $\mathbf{x}$ and proceed with the next one (see the bold type line in Fig. 2). Indeed, since the objective function can only increase with the number $i$ of processed scenarios, the current $\mathbf{x}$ could only lead to an objective larger than $B$.

This significant short-cut of the enumerative search is nothing but an application of the branch-and-bound technique $[10]$.

Furthermore, when using the $L_{\infty}$ objective, the branchand-bound algorithm itself can be improved by a scenario 
set $B$ to an arbitrary large value

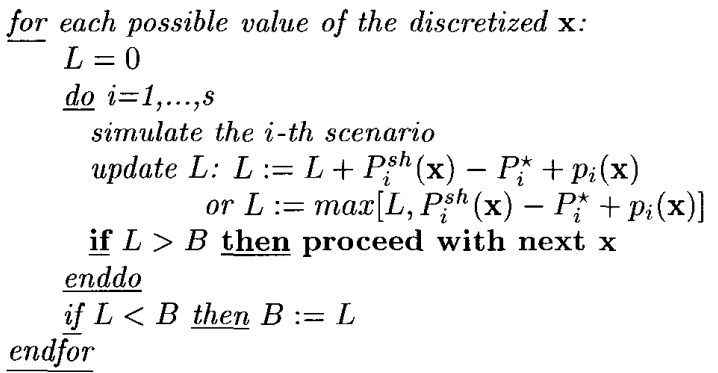

the global optimum corresponds to $B$

Fig. 2. purely enumerative and branch-and-bound approaches

reordering strategy. The latter consists in memorizing during the optimization how many times each scenario has lead to a broken enumeration. Once the optimal parameters have been found, the different scenarios are ranked by decreasing order of the break frequency. The top ranked scenarios can be considered to be the most constraining for the optimization, i.e. to lead the most frequently to objectives greater than $B$. Hence, by enumerating these scenarios first, additional savings in computing times can be expected when the algorithm will be re-executed using the same set of training scenarios. This will be for example the case when the transmission system is modified or when the controller settings are updated according seasonal changes in load. The scenario ranking has to be updated after each optimization. "

The branch-and-bound approach and the reordering strategy can be used when optimizing any of the three types of controllers defined in section II.

In the case of the FSFD controller, the optimization problem can be formulated as a tree exploration and the branch-and-bound algorithm can be further improved as detailed in [11].

\section{RESULTS ON HYDRO-QUEBEC SYSTEM}

\section{A. Voltage stability of the Hydro-Québec system}

The Hydro-Québec (HQ) system is characterized by great distances (more than $1000 \mathrm{~km}$ ) between the large hydro generation areas (James Bay, Churchill Falls and Manic-Outardes) and the main load center (around Montréal and Québec City). Accordingly, the company has developed an extensive $735-\mathrm{kV}$ transmission system, whose lines are located along two main axes. This system is voltage stability limited in the South, near loads.

In the recent years, Hydro-Québec has undertaken a major program to upgrade the reliability of its transmission system. In particular a defence plan is being deployed against extreme contingencies [12]. This includes generation rejection and remote load shedding, automatic shunt reactor switching, underfrequency load shedding and in a near future, undervoltage load shedding.

Beside static var compensators and synchronous condensers, the automatic shunt reactor switching devices, known under the French acronym MAIS, play an important role in voltage stability [13]. These devices, in operation since early 1997, are now available in twenty-two $735-\mathrm{kV}$ substations and control a large part of the total 25,500 Mvar shunt compensation. Each MAIS relies on the local voltage, the coordination between substations being performed through the switching delays.

Preventive security assessment is also a major concern. Presently, secure operation limits are determined in operational planning, with the help of ASTRE, the Quasi Steady-state (QSS) simulation program developed at the University of Liège [4], [14]. This fast tool has been used in the present study. The corresponding model includes around 550 buses, 100 generators and 230 LTCs, for a total load of about $33,000 \mathrm{MW}$.

\section{B. Training scenarios}

The study reported in this paper involves 8 system configurations, summarized in Table 1.

Table 1. Configurations considered in the training scenarios

\begin{tabular}{|c|c|c|c|c|}
\hline $\begin{array}{c}\text { configur- } \\
\text { ation }\end{array}$ & $\begin{array}{c}\text { 735-kV lines } \\
\text { out of service }\end{array}$ & $\begin{array}{c}\text { synchronous } \\
\text { condensers }\end{array}$ & $\begin{array}{c}\text { MAIS } \\
\text { devices }\end{array}$ & $\begin{array}{c}\text { scenarios } \\
\text { no }\end{array}$ \\
\hline \hline A & 0 & 6 & 5 & $1 \rightarrow 6$ \\
B & 1 & 6 & 9 & $7 \rightarrow 8$ \\
C & 1 & 6 & 8 & 9 \\
D & 1 & 8 & 16 & $10 \rightarrow 14$ \\
E & 1 & 8 & 14 & $15 \rightarrow 20$ \\
F & 4 & 8 & 16 & $21 \rightarrow 25$ \\
G & 3 & 8 & 14 & $26 \rightarrow 31$ \\
H & -3 & 8 & 14 & $32 \rightarrow 36$ \\
\hline
\end{tabular}

Tables 1 and 2 detail the 36 scenarios finally selected. They involve N-1, N-2 and N-3 contingencies, respectively. In accordance with the standard operating rules, the system is stable following any N-1 incident. The MAIS devices can be used to this purpose. In each unstable scenario, the

Table 2. Description of the 36 training scenarios

\begin{tabular}{|c|c|c|r||c|c|c|r|}
\hline No & incid. & \multicolumn{2}{|c|}{$P_{i}^{\star}(\mathrm{MW})$} & No & incid. & \multicolumn{2}{|c|}{$P_{i}^{\star}(\mathrm{MW})$} \\
& type & Norm. & MAIS & & type & Norm. & MAIS \\
\hline 1 & $\mathrm{~N}-1$ & 0 & 0 & 19 & $\mathrm{~N}-2$ & 890 & 480 \\
2 & $\mathrm{~N}-1$ & 0 & 0 & 20 & $\mathrm{~N}-2$ & 890 & 610 \\
3 & $\mathrm{~N}-2$ & 1090 & 1090 & 21 & $\mathrm{~N}-1$ & 0 & 0 \\
4 & $\mathrm{~N}-2$ & 460 & 360 & 22 & $\mathrm{~N}-2$ & 0 & 0 \\
5 & $\mathrm{~N}-2$ & 80 & 30 & 23 & $\mathrm{~N}-2$ & 1110 & 940 \\
6 & $\mathrm{~N}-3$ & 1520 & 1500 & 24 & $\mathrm{~N}-2$ & 860 & 340 \\
7 & $\mathrm{~N}-1$ & 0 & 0 & 25 & $\mathrm{~N}-2$ & 620 & 130 \\
8 & $\mathrm{~N}-2$ & 200 & 10 & 26 & $\mathrm{~N}-1$ & 0 & 0 \\
9 & $\mathrm{~N}-1$ & 0 & 0 & 27 & $\mathrm{~N}-1$ & 0 & 0 \\
10 & $\mathrm{~N}-1$ & 0 & 0 & 28 & $\mathrm{~N}-2$ & 30 & 0 \\
11 & $\mathrm{~N}-1$ & 0 & 0 & 29 & $\mathrm{~N}-2$ & 1790 & 1650 \\
12 & $\mathrm{~N}-2$ & 0 & 0 & 30 & $\mathrm{~N}-2$ & 880 & 770 \\
13 & $\mathrm{~N}-2$ & 740 & 490 & 31 & $\mathrm{~N}-2$ & 720 & 310 \\
14 & $\mathrm{~N}-2$ & 340 & 0 & 32 & $\mathrm{~N}-1$ & 0 & 0 \\
15 & $\mathrm{~N}-1$ & 0 & 0 & 33 & $\mathrm{~N}-1$ & 0 & 0 \\
16 & $\mathrm{~N}-1$ & 0 & 0 & 34 & $\mathrm{~N}-2$ & 240 & 30 \\
17 & $\mathrm{~N}-2$ & 0 & 0 & 35 & $\mathrm{~N}-2$ & 720 & 640 \\
18 & $\mathrm{~N}-2$ & 0 & 0 & 36 & $\mathrm{~N}-2$ & 580 & 100 \\
\hline
\end{tabular}

best load shedding location has been identified. Therefrom, 
a common ranking of load buses has been set up. Using this bus ranking, the minimal amount of load shedding $P_{i}^{\star}$ required to stabilize the system has been determined in the 19 unstable scenarios. The values, computed with an accuracy of $10 \mathrm{MW}$, are given in Table 2 . The most severe incident requires to shed load at 8 buses.

In this determination, we supposed that reactors are tripped:

- either all together just after the disturbance, as discussed in section III.a (results given in the "MAIS" column);

- or by the MAIS controllers, as implemented presently in the HQ system (results given in the "Norm." column).

The scenarios have been chosen according to the following guidelines:

- the training set includes 17 stable scenarios in order to train the protection not to act in stable cases;

- on the other hand, the nonzero values of $P_{i}^{\star}$ range rather uniformly in the [0. 1790] MW interval, between the marginally and the severely unstable cases.

The measured signal $V$ is the average voltage over five $735-\mathrm{kV}$ buses in the Montréal area. Requirements 1, 2 and 3 of Section III.C have been taken into account. However, in accordance with Hydro-Québec planning rules, the 3rd requirement has been amended by allowing some (hopefully small) load shedding to take place after a stable but severe incident. The N-2 scenarios No 12, 17, 18 and 22 are concerned. The latter are handled as unstable scenarios with $P_{i}^{\star}=0$ in (7) and (8).

\section{Preliminary choices of controller parameters}

In order to obtain a good synchronization with MAIS whose settings are typically in the range [0.965 $0.97 \mathrm{pu}]$, the $V_{1}^{\text {min }}$ parameter of (1) and the $V^{\text {min }}$ parameters of (4-6) were adjusted to $0.95 \mathrm{pu}$. Moreover, this value guarantees that the controllers will not act in the stable situations of Table 2, except for scenario 10 whose voltage falls below $0.93 \mathrm{pu}$ at the beginning of the simulation. In the case of $2-$ or 3-rule controllers, the thresholds relative to rules 2 and 3 were adjusted to 0.93 and 0.91 pu respectively, in order to act before the amount to be shed becomes prohibitive and also to minimize the customers'trouble.

For each kind of controller, the lower bound on each delay is fixed to $3 \mathrm{~s}$ in order to distinguish a voltage instability from a temporary undervoltage. On the other hand, the lower bound on load shedding is the minimum amount that can be tripped by opening distribution feeders, which corresponds to about $50 \mathrm{MW}$, while the upper bound has been limited to $800 \mathrm{MW}$ to avoid excessive load shedding steps.

As regards the other parameters, the total number of setting combinations is given in Table 3 for each type of controller. Taking into account that each combination has to be tested over at most $s$ scenarios, these figures confirm the huge complexity of the combinatorial optimization.
Table 3. Total number of combinations for each controller

\begin{tabular}{|c|c||c|c|}
\hline $\begin{array}{c}\text { controller } \\
\text { type }\end{array}$ & $\begin{array}{c}\text { total nb } \\
\text { of settings }\end{array}$ & $\begin{array}{c}\text { controller } \\
\text { type }\end{array}$ & $\begin{array}{c}\text { total nb } \\
\text { of settings }\end{array}$ \\
\hline 2-rule FSFD & 6864 & VSVD & 6300 \\
3-rule FSFD & 95550 & FSVD coord & 60480 \\
FSVD & 5040 & VSFD coord & 53760 \\
VSFD & 4480 & VSVD coord & 75600 \\
\hline
\end{tabular}

\section{Results and discussion}

Table 4 (resp. 5) shows for each type of controller the optimal value of the $L_{1}$ (resp. $L_{\infty}$ ) objective as well as the corresponding computing time. For information only, the value of the other (non optimized) objective is given in parentheses in the second column.

As can be seen, optimizing the $L_{1}$ objective is much more time consuming, whatever the type of controller. The reason is that for the same vector $\mathbf{x}$ of settings, a larger amount of time is needed before the $L_{1}$ objective becomes greater than $B$ in the branch-and-bound algorithm, since $B$ relates to the sum of discrepancies. Moreover, the reordering strategy cannot be used when considering this objective, while this heuristics allows to significantly improve the performances of the basic algorithm when optimizing the $L_{\infty}$ objective.

As expected, the VSVD controllers require less computing time than the FSFD ones since they involve less parameters (see end of section II.A).

For the same reason, the coordinated controllers are more computationally demanding than the uncoordinated ones.

Table 4. Optimization results when using the $L_{\infty}$ objective
\begin{tabular}{|c|c|c|}
\hline $\begin{array}{c}\text { type of } \\
\text { controller }\end{array}$ & $\begin{array}{c}L_{\infty}\left(L_{1}\right) \text { obj. } \\
\text { function (in MW) }\end{array}$ & $\begin{array}{c}\text { computing time } \\
\text { (hours) }\end{array}$ \\
\hline 2-rule FSFD & $320(128)$ & 5.6 \\
3-rule FSFD & $290(116)$ & 15.3 \\
FSVD & $340(130)$ & 4.5 \\
VSFD & $280(118)$ & 5.4 \\
VSVD & $280(112)$ & 8 \\
FSVD coord & $300(67)$ & 93 \\
VSFD coord & $305(74)$ & 113 \\
VSVD coord & $304(80)$ & 122 \\
\hline
\end{tabular}

\begin{tabular}{|c|c|c|}
\hline $\begin{array}{c}\text { type of } \\
\text { controller }\end{array}$ & $\begin{array}{c}L_{1}\left(L_{\infty}\right) \circ b j . \\
\text { function (in MW) }\end{array}$ & $\begin{array}{l}\text { computing time } \\
\text { (hours) }\end{array}$ \\
\hline 2-rule FSFD & $115(380)$ & 50 \\
\hline 3-rule FSFD & $114(460)$ & 555 \\
\hline FSVD & $124(370)$ & 31 \\
\hline VSFD & $113(340)$ & 45 \\
\hline VSVD & $112(280)$ & 64.6 \\
\hline FSVD coord & $67(300)$ & 406 \\
\hline VSFD coord & $71(498)$ & 516 \\
\hline VSVD coord & $68(499)$ & 724 \\
\hline
\end{tabular}

Figure 3 shows, in each unstable scenario, the total load shed by the coordinated and uncoordinated VSFD controllers, respectively. Both are based on the $L_{\infty}$ objective. As expected, the coordinated controller leads to less load shedding. The uncoordinated controller gives better results only in scenarios 3 and 6 . It can be checked from Table 2 that in these two scenarios the $P_{i}^{\star}$ value does not depend on the time sequence of shunt reactor trippings. 


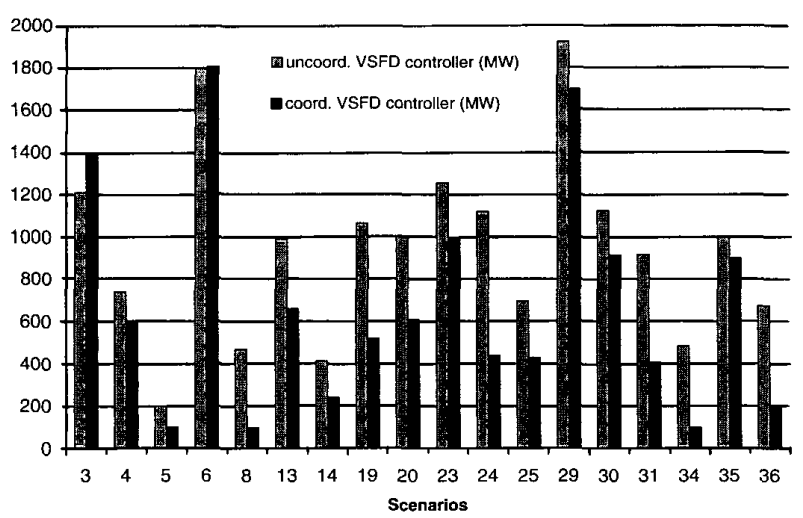

Fig. 3. VSFD and coord. VSFD controllers performances

To summarize, the coordinated controllers have better performances but require more computing time. An interesting trade-off would consist in working with uncoordinated VSVD controllers. Indeed, although they are less effective than their coordinated counterpart, they are the best among the uncoordinated schemes.

Coming back to the results of Tables 4 and 5, it appears that the coordinated controllers are characterized by lower $L_{1}$ but higher $L_{\infty}$ objective values. In fact, the $L_{\infty}$ objective value of a coordinated controller cannot be compared with that of an uncoordinated one. Indeed, the $L_{\infty}$ norm only relates to the maximum discrepancy between $P_{i}^{s h}$ and $P_{i}^{\star}$. Now, the $P_{i}^{\star}$ values are different for the two types of controllers (see Table 2). The same holds for the $L_{1}$ objective.

A comparison would be possible if the dependence on $P_{i}^{\star}$ was removed from the objective function. This can be done for the $L_{1}$ norm (7), as shown hereafter. Considering that, at the optimum, the penalties are zero, the correponding $L_{1}$ objective can be rewritten as $\frac{1}{s} \sum_{i}\left[P_{i}^{s h}(\mathbf{x})-P_{i}^{\star}+p_{i}(\mathbf{x})\right]=$ $\frac{1}{s} \sum_{i} P_{i}^{s h}(\mathbf{x})-C$ where $C$ is constant. Thus, optimizing $\frac{1}{s} \sum_{i} P_{i}^{s h}(\mathbf{x})$ will lead to the same results as optimizing the original $L_{1}$ objective. The same does not hold for the $L_{\infty}$ norm, since using $\max P_{i}^{s h}(\mathbf{x})$ as an objective would lead to concentrate on the most severe scenario only, which is not the case with the original criterion (8).

\section{CONCLUSIONS AND PERSPECTIVES}

In this paper, three types of undervoltage load shedding controllers have been compared. A methodology to optimize their parameters has been described and illustrated on a rather complex example. The VSVD controller seems to be the most appropriate among the uncoordinated schemes. They are however less effective than coordinated controllers managing both load shedding and automatic shunt reactor switching. On the other hand, the latter require to collect measurements from, and send controls to remote locations. The higher communication complexity has to be taken into account when assessing the overall protection reliability.

Obviously, several aspects remain to be investigated. We quote hereafter two of them.

For the sake of simplicity, we considered here that the amount of load shed by VSVD controllers can be arbitrary adjusted to any value between $\Delta P_{\min }$ and $\Delta P_{\max }$ according to the linear relationship (3). In real-life systems, however, load is shed by opening circuit breakers. This technical constraint could be taken into account by imposing the amount of shed load to vary by steps in between $\Delta P_{\text {min }}$ and $\Delta P_{\max }$. It would be interesting to evaluate how this constraint influences the performances of the VSFD and VSVD controllers.

From the optimization point of view, a possible drawback of the proposed method is the risk of training set overfitting. This could be solved by considering very large training sets involving many stable and unstable scenarios. However, due to the combinatorial nature of the problem, the computing time would become prohibitive, in spite of the effectiveness of the branch-and-bound method.

An interesting trade-off between computing time, overfitting and effectiveness could be obtained by carrying out the optimization in two steps. First, all the controllers yielding an objective function below some predefined level could be identified, using a limited number of scenarios taken from the large training set. In a second step, these controllers could be tested on the remaining scenarios in order to find the design leading to the best average performance on the whole training set.

\section{REFERENCES}

[1] Final report of TF 38.02.19, "System protection schemes in power networks," Tech. Rep., CIGRE, to appear in 2001.

[2] A. Moshref P. Kundur, G. K. Morison, "Measures to improve power system security in the new competitive market environment," in VIIth Sepope Proceedings, Curitiba, 2000.

[3] C. W. Taylor, Power System Voltage Stability, EPRI Power System Engineering series. 1994.

[4] C. Vournas T. Van Cutsem, Voltage Stability of Electric Power Systems, Kluwer Academic Publishers, Boston, 1998.

[5] D. Hill S. Arnborg, G. Andersson and I. Hiskens, "On undervoltage load shedding in power systems," in Electric Power and Energy Systems, 1997, vol. 19, pp. 141-149.

[6] J.T. Saraiva J.R. Ferreira, J.A. Peças Lopes, "Identification of preventive control procedures to avoid voltage collapse using genetic algorithms," in 13th PSCC Proceedings, Trondheim, 1999, pp. $463-469$.

[7] C. W. Taylor, "Concepts of undervoltage load shedding for voltage stability," IEEE Trans. on Power Delivery, vol. 7, pp. 480$488,1992$.

[8] M. Stephens S. Kolluri, K. Tinnium, "Design and operating experience with fast acting load shedding scheme in the entergy system to prevent voltage collapse," in IEEE Power Engineering Society Winter Meeting.

[9] T. Van Cutsem C. Moors, "Determination of optimal load shedding against voltage instability," in 13th PSCC Proceedings, Trondheim, 1999, pp. 993-1000.

[10] W. Pulley Blank W. Cook, W. Cunningham and A. Schrijver, Combinatorial Optimization, Wiley-Interscience, 1998.

[11] T. Van Cutsem C. Moors, D. Lefebvre, "Combinatorial optimization approaches to the design of load shedding schemes against voltage instability," in 21th North American Power Symposium, Waterloo, Canada, 2000.

[12] G. Scott G. Trudel, S. Bernard, "Hydro-québec's defence plan against extreme contingencies," in paper PE-211-PWRS-0-061998 presented at the IEEE PES Summer Meeting, San Diego, July 1998.

[13] G. Scott S. Bernard, G. Trudel, "A 735-kv shunt reactors automatic switching system for hydro-qubec network," IEEE Trans. on Power Systems, vol. 11, pp. 2024-2030, 1996.

[14] R. Mailhot T. Van Cutsem, "Validation of a fast voltage stability analysis method on the hydro-qubec system," IEEE Trans. on Power Systems, vol. 12, pp. 282-292, 1997. 